\title{
010 PP COMMUNITY LINKAGES: A DRIVER OF EFFECTIVE IMMUNIZATION? - A PARTICIPATORY EVALUATION AND ACTION RESEARCH
}

N Akwataghibe, ${ }^{1 *}$ E Ogunsola, ${ }^{2}$ O Popoola, ${ }^{3}$ A Emeji, ${ }^{4}$ M Dieleman ${ }^{1} .{ }^{1}$ Royal Tropical Institute, The Netherlands; Athena Institute, Faculty of Earth and Life Sciences, VU University Amsterdam, The Netherlands; ${ }^{2}$ Primary Health Care Development Board (PHCDB) Ogun State, Nigeria; ${ }^{3}$ Department of Community Medicine, University College Hospital, University of Ibadan, Nigeria; ${ }^{4}$ School of Community Health and Policy (SCHP), Morgan State University, USA

10.1136/bmjopen-2017-016492.28

Background: In 2005, Nigeria adopted the Reach Every Ward (REW) strategic approach to improve vaccination coverage for 
children of 0-23 months. Ogun state has coverage of up to $107 \%$ in 12 of its 20 local government areas (LGA). Conversely, there are pockets of unimmunized children across eight LGAs with the highest burden in Remo-North (23\%). The exact factors responsible for this trend are yet to be determined.

Objective: A participatory evaluation and action research (PAR) aims through iterative processes of reflection and action, between communities, health workers and local authorities to provide context specific solutions to barriers to access and use of immunization services in Remo-North. A formative evaluation assesses the relevance, efficiency and effectiveness of the PAR.

Methods: Situational analysis was carried out at baseline, results of which were validated by community members, health workers and local government officials and used in an interactive learning process through dialogues between these groups, facilitated by the researchers. Joint Action Plans for change were developed and are being implemented in a first action phase of 4 months. The circle will repeat in a second round of dialogues/ action phase. After one year, at end-line, the key components of change to be assessed are improved community knowledge and awareness of the REW strategy; and consensus on challenges and solutions regarding immunization among the three groups. Additionally, demonstrated ownership and responsibility for change among these groups will be assessed in order to identify the potential for sustained improvement of access and utilization of immunization services. 